\title{
ON THE UNIQUE REPRESENTATION OF FAMILIES OF SETS
}

\author{
SU GAO, STEVE JACKSON, MIKLÓS LACZKOVICH, AND R. DANIEL MAULDIN
}

\begin{abstract}
Let $X$ and $Y$ be uncountable Polish spaces. $A \subset X \times Y$ represents a family of sets $\mathcal{C}$ provided each set in $\mathcal{C}$ occurs as an $x$-section of $A$. We say that $A$ uniquely represents $\mathcal{C}$ provided each set in $\mathcal{C}$ occurs exactly once as an $x$-section of $A$. $A$ is universal for $\mathcal{C}$ if every $x$-section of $A$ is in $\mathcal{C}$. $A$ is uniquely universal for $\mathcal{C}$ if it is universal and uniquely represents $\mathcal{C}$. We show that there is a Borel set in $X \times R$ which uniquely represents the translates of $\mathbb{Q}$ if and only if there is a $\Sigma_{2}^{1}$ Vitali set. Assuming $V=L$ there is a Borel set $B \subset \omega^{\omega}$ with all sections $F_{\sigma}$ sets and all non-empty $K_{\sigma}$ sets are uniquely represented by $B$. Assuming $V=L$ there is a Borel set $B \subset X \times Y$ with all sections $K_{\sigma}$ which uniquely represents the countable subsets of $Y$. There is an analytic set in $X \times Y$ with all sections $\Delta_{2}^{0}$ which represents all the $\Delta_{2}^{0}$ subsets of $Y$, but no Borel set can uniquely represent the $\Delta_{2}^{0}$ sets. This last theorem is generalized to higher Borel classes.
\end{abstract}

\section{INTRODUCTION}

Throughout the paper, $X, Y$ will denote uncountable Polish spaces. For $A \subseteq$ $X \times Y$, denote the $x$-section of $A$ by $A_{x}$, i.e., let

$$
A_{x}=\{y \in Y \mid(x, y) \in A\} .
$$

We also call $A_{x}$ a section of $A$. By a pointclass we mean a collection $\boldsymbol{\Gamma}$ of subsets of Polish spaces which is closed under inverse images by continuous functions between Polish spaces.

Definition 1.1. A class of sets $\mathcal{C} \subseteq \mathcal{P}(Y)$ is said to be represented by $A$ if

$$
\mathcal{C} \subseteq\left\{A_{x} \mid x \in X\right\}
$$

The class $\mathcal{C}$ is said to be uniquely represented by $A$ if for every set $C \in \mathcal{C}$ there is a unique $x \in X$ such that $C=A_{x}$. We say $A$ is universal for a class of nonempty sets $\mathcal{C}$ provided $A$ represents $\mathcal{C}$ and every non-empty section $A_{x}$ is in $\mathcal{C}$. We say $A$ is uniquely universal for $\mathcal{C}$ if $A$ is universal for $\mathcal{C}$ and uniquely represents $\mathcal{C}$.

In this paper we consider various problems concerning the existence of sets providing unique representations for certain families of sets. The various properties we consider can be abstracted into the following definition.

Received by the editors April 28, 2005 and, in revised form, December 28, 2005.

2000 Mathematics Subject Classification. Primary 54H05, 22F05; Secondary 54E35, 03E75.

Key words and phrases. Unique representations, uniquely universal sets, Vitali sets, scattered sets.

The second author was supported by NSF grant DMS 0097181.

The third author thanks the mathematics department of UNT for supporting his research visit.

The fourth author was supported by NSF grant DMS 0400481. 
Definition 1.2. Let $\Gamma_{1}$ be a pointclass, and $\mathcal{C}_{2}, \mathcal{C}_{3} \subseteq \mathcal{P}(Y)$. We say that the unique representation property $U\left(\boldsymbol{\Gamma}_{1}, \mathcal{C}_{2}, \mathcal{C}_{3}\right)$ holds if there is an $A \subseteq X \times Y, A \in \boldsymbol{\Gamma}_{1}$, such that all sections $A_{x}$ of $A$ are in $\mathcal{C}_{2}$, and $A$ uniquely represents $\mathcal{C}_{3}$. We define the representation property $R\left(\boldsymbol{\Gamma}_{1}, \mathcal{C}_{2}, \mathcal{C}_{3}\right)$ in the same manner except we require in the last clause only that $A$ represents $\mathcal{C}_{3}$.

General Problem. For which $\boldsymbol{\Gamma}_{1}, \mathcal{C}_{2}, \mathcal{C}_{3}$ does $U\left(\boldsymbol{\Gamma}_{1}, \mathcal{C}_{2}, \mathcal{C}_{3}\right)\left(\right.$ or $\left.R\left(\boldsymbol{\Gamma}_{1}, \mathcal{C}_{2}, \mathcal{C}_{3}\right)\right)$ hold?

We emphasize that $\mathcal{C}_{2}, \mathcal{C}_{3}$ need not be pointclasses. We will consider below cases where they correspond to the collection of countable sets, or to the $K_{\sigma}$ sets.

Note that the statement that the pointclass $\boldsymbol{\Gamma}$ has a universal set is just $R(\boldsymbol{\Gamma}, \boldsymbol{\Gamma}, \boldsymbol{\Gamma})$. Of course, a self-dual pointclass such as $\boldsymbol{\Delta}_{1}^{1}$ cannot have a universal set, but an old theorem of Sierpinski says that there is an analytic set which is universal for the Borel sets, that is, $R\left(\boldsymbol{\Sigma}_{1}^{1}, \boldsymbol{\Delta}_{1}^{1}, \boldsymbol{\Delta}_{1}^{1}\right)$.

The genesis of our work is the following still unsolved problem of the fourth author.

Problem 1.3. Is there a Borel subset of $X \times Y$ which is uniquely universal for the class of $K_{\sigma}$ subsets of $Y$ ? Or even, is there a Borel set which uniquely represents the $K_{\sigma}$ sets?

One of the original motivations for the problem comes from a question concerning the "graphs of multifunctions" and the complexity of the $\in$ relation. We comment on this relationship by noting the next theorem.

Theorem 1.4. Let $X$ be an uncountable Polish space. The following statements are equivalent:

(i) There is a standard Borel structure $\mathcal{B}$ on $K_{\sigma}(X)$ such that the $\in$ relation is in the $\sigma$-algebra $\mathcal{B}(X) \times \mathcal{B}$.

(ii) There is a Borel set $U$ in $X \times X$ which is uniquely universal for the family $K_{\sigma}(X)$.

Proof. Assume (i) holds. Let $\psi$ be a $(\mathcal{B}(X), \mathcal{B})$ isomorphism of $X$ onto $K_{\sigma}(X)$. Let $U=\{(x, y): y \in \psi(x)\}$. Note that $U$ is uniquely universal for $K_{\sigma}(X)$ and $U=\operatorname{proj}_{12}(W)$ where $W=\{(x, y, K): y \in K \wedge \psi(x)=K\}$. Since $W$ is a Borel set and proj $_{12}$ is 1-to- 1 on $W, U$ is a Borel set.

Secondly, assume (ii) holds. Let $D=\operatorname{proj}_{1}(U)$. Then by Saint-Raymond's theorem [14], $D$ is a Borel subset of $X$. Define $\gamma: D \mapsto K_{\sigma}(X)$ by $\gamma(x)=U_{x}$. Let $\mathcal{B}=\{\gamma(E): E$ is a Borel subset of $D\}$. Then $\mathcal{B}$ is a standard Borel structure on $K_{\sigma}(X)$. Also, $\in=\operatorname{proj}_{23}(G)$, where $G=\left\{(x, y, K) \in X \times X \times K_{\sigma}(X): K=\right.$ $\gamma(x) \wedge(x, y) \in U\}$ and $\operatorname{proj}_{23}$ is 1-to-1 on the Borel set $G$.

Of course, we can ask in some generality whether there is a natural Borel structure on various families of sets. We can consider "natural" to mean that various operations and relations must be measurable, the most basic relation being the "belongs to" relation. In particular, we can ask the same question for the $F_{\sigma}$ sets.

Problem 1.5. Is there a Borel subset of $X \times Y$ which is uniquely universal (or even uniquely represents) the class of $F_{\sigma}\left(=\Sigma_{2}^{0}\right)$ subsets of $Y$ ?

Both of these problems remain unsolved. In this paper we obtain some partial results about them and related problems.

We fix a recursive bijection $(n, m) \rightarrow\langle n, m\rangle$ between $\omega \times \omega$ and $\omega$. We let $n \rightarrow\left((n)_{0},(n)_{1}\right)$ denote the inverse of this map. This extends to a homeomorphism 
between $\omega^{\omega}$ and $\left(\omega^{\omega}\right)^{\omega}$ in a standard way, namely, for $\left(x_{i}\right)_{i \in \omega} \in\left(\omega^{\omega}\right)^{\omega}$, let $y=$ $\left\langle x_{i}\right\rangle_{i \in \omega}$ be the real coding them defined by $y(n)=x_{(n)_{0}}\left((n)_{1}\right)$. For $x \in \omega^{\omega}$, let $C(x)=\left\{(x)_{0},(x)_{1}, \ldots\right\}$ be the countable set coded by $x\left(\right.$ so $\left.(x)_{n}(m)=x(\langle n, m\rangle)\right)$. We abuse notation slightly and also use $(x, y) \rightarrow\langle x, y\rangle$ and $x \rightarrow\left((x)_{0},(y)_{0}\right)$ to denote homeomorphisms between $\left(\omega^{\omega}\right)^{2}$ and $\omega^{\omega}$.

With the above notation it is easy to find Borel sets in $\omega^{\omega} \times \omega^{\omega}$ (or $\mathbb{R} \times \mathbb{R}$ ) which represent the class of all non-empty countable subsets of $\omega^{\omega}$ (or $\mathbb{R}$ ).

Remark 1.6. We note that in most cases the Polish space $X$ above is immaterial, and $Y$ can in some cases be replaced by $\omega^{\omega}$. To see the first statement, suppose $X_{1}, X_{2}$ are uncountable Polish spaces and let $\phi: X_{2} \rightarrow X_{1}$ be a Borel (in fact $\boldsymbol{\Delta}_{3}^{0}$ ) bijection. If $A_{1} \subseteq X_{1} \times Y$, let $A_{2}$ be defined by $A_{2}(x, y) \leftrightarrow A_{1}(\phi(x), y)$. If $\boldsymbol{\Gamma}$ is closed under Borel preimages (e.g., $\boldsymbol{\Gamma}=\boldsymbol{\Delta}_{1}^{1}, \boldsymbol{\Sigma}_{1}^{1}$ ), then $A_{1} \in \boldsymbol{\Gamma}$ iff $A_{2} \in \boldsymbol{\Gamma}$ and $A_{1}$, $A_{2}$ have the same sections; thus $A_{1}$ is uniquely universal for (or uniquely represents, etc.) a collection $\mathcal{C} \subseteq \mathcal{P}(Y)$ iff $A_{2}$ is.

For the second statement, first note that if $\mathcal{C}$ is defined for all Polish spaces and is closed under Borel bijections (e.g., $\mathcal{C}=$ countable sets), then there is a Borel (or analytic, etc.) set in $X \times Y$ representing (or uniquely representing, or uniquely universal for) $\mathcal{C}$ iff there is such a set in $X \times \omega^{\omega}$. This follows immediately by considering a Borel bijection between $Y$ and $\omega^{\omega}$. In particular, there is a Borel set in $X \times Y$ with all sections countable and uniquely representing the countable (or non-empty countable) sets in $Y$ iff there is such a set in $\omega^{\omega} \times \omega^{\omega}$.

Secondly, suppose $Y$ is an uncountable Polish space. There is a countably infinite set $C=\left\{y_{n}\right\}_{n \in \omega} \subseteq Y$ such that $Y \backslash C$ is the continuous one-to-one image of $\omega^{\omega}$, say by $\phi: \omega^{\omega} \rightarrow(Y \backslash C)$. If $A_{1} \subseteq \omega^{\omega} \times \omega^{\omega}$, define $A_{2} \subseteq \omega^{\omega} \times Y$ by

$$
A_{2}(x, y) \leftrightarrow\left[(y \notin C) \wedge A_{1}\left((x)_{0}, \phi^{-1}(y)\right)\right] \vee \exists n\left[y=y_{n} \wedge(x)_{1}(n)=0\right] .
$$

Clearly $A_{2}$ will be Borel (or analytic, etc.) iff $A_{1}$ is. Suppose $\mathcal{C}_{2}$ is a collection which is defined for both $Y$ and $\omega^{\omega}$ and is closed under continuous one-to-one images and also unions with countable sets (for example, $\mathcal{C}_{2}=K_{\sigma}$ ). Suppose $\mathcal{C}_{3}$ is a collection defined for $Y$ which is closed under unions and differences with countable sets, that is, if $A \in \mathcal{C}$, then so is $A \cup C$ and $A \backslash C$ whenever $C$ is countable (for example, $\mathcal{C}_{3}=$ countable sets). Then if $A_{1}$ is a Borel (or analytic, etc.) set witnessing $U\left(\boldsymbol{\Gamma}_{1}, \mathcal{C}_{2}, \mathcal{C}_{3}\right)$ for $X \times \omega^{\omega}$, then $A_{2}$ witnesses $U\left(\boldsymbol{\Gamma}_{1}, \mathcal{C}_{2}, \mathcal{C}_{3}\right)$ for $X \times Y$. So, for example, there is a Borel set in $X \times Y$ with all sections $K_{\sigma}$ which uniquely represents the countable sets iff there is such a set in $\omega^{\omega} \times \omega^{\omega}$.

On the other hand, it is not immediately clear if the space $Y$ is relevant in problems 1.3, 1.5 .

We employ a standard coding of Borel sets in a Polish space $X$. In some straightforward manner we view every $w \in \omega^{\omega}$ as coding a tree $T \subseteq(\omega \times \omega)^{<\omega}$ together with a function which assigns to the terminal nodes $s$ of $T$ an integer $n_{s}$ which codes a basic open set $N_{n_{s}}$ in $X$. We say $w$ is a Borel code if the tree $T$ is wellfounded, in which case $w$ determines a Borel subset $B(w)$ of $X$ in the usual manner [for $s$ terminal in $T$, associate to $s$ the set $B(w, s)=N_{n_{s}}$, and for $s$ non-terminal let $B(w, s)=X \backslash \bigcup\left\{B\left(x, s^{\urcorner} n\right) \mid s^{\urcorner} n \in T\right\}$. Let $\left.B(w)=B(w, \emptyset)\right]$.

In Section 2 we consider first the existence of a Borel set which uniquely represents all the translates of $\mathbb{Q}$. The existence of such a set turns out to be equivalent to the existence of a $\Sigma_{2}^{1}$ Vitali set. Furthermore, we show there is no Borel set with countable sections which uniquely represents the translates of $\mathbb{Q}$. 
In Section 3 we give another proof of the known result (cf. [1]) that there is no Borel set with countable sections uniquely representing the countable sets (that is, $U\left(\boldsymbol{\Delta}_{1}^{1}, \mathcal{C}_{\text {ctbl }}, \mathcal{C}_{\text {ctbl }}\right)$ fails, where $\mathcal{C}_{\text {ctbl }}$ is the class of countable sets). Although this result follows from the results of Section 2, we give a different proof using a forcing argument similar to one in [5], which gives some extra information. By contrast, it is a theorem of Becker [1] that it is consistent to have an analytic set with countable sections which uniquely represents the countable sets (i.e., $U\left(\boldsymbol{\Sigma}_{1}^{1}, \mathcal{C}_{\text {ctbl }}, \mathcal{C}_{\text {ctbl }}\right)$ is consistent). We show that it is consistent that there is a Borel set with $F_{\sigma}$ sections which uniquely represents the countable or even $K_{\sigma}$ sets. These results serve as motivation and a warm-up for the main result in Section 4.

In Section 4 we show that it is consistent to have a Borel set with $K_{\sigma}$ sections which uniquely represents the countable sets. This result seems to be "half-way" between the negative result of the previous paragraph and problem 1.3.

In Section 5 we show that there is no Borel set with $\boldsymbol{\Delta}_{\alpha+1}^{0}$ sections which even represents the $\boldsymbol{\Delta}_{\alpha+1}^{0}$ sets.

As we note below, unique representability results fail in the presence of large cardinal (or determinacy) axioms. For example, assuming $\boldsymbol{\Pi}_{1}^{1}$ determinacy there is no Borel set uniquely representing the countable sets, which gives a negative answer to the questions in problems $1.3,1.5$. Thus, the question is whether such results are consistently true or refutable in ZFC.

\section{Unique REPRESEntations of the translates of $\mathbb{Q}$}

Lemma 2.1. Let $B$ be a Borel subset of $X \times \mathbb{R}$. Then $C=C(B)=\{x$ : $B_{x}$ is a translate of $\left.Q\right\}$ is $\Pi_{1}^{1}$. If, in addition, every section of $B$ is countable, then $C$ is a Borel subset of $X$.

Proof. Notice that

$$
X \backslash C=\operatorname{proj}_{1}(E) \cup \operatorname{proj}_{1}(F),
$$

where

$$
(x, y, z) \in E \Leftrightarrow(x, y),(x, z) \in B \wedge y-z \notin \mathbb{Q}
$$

and

$$
(x, y) \in F \Leftrightarrow(x, y) \in B \wedge \exists q \in \mathbb{Q}[(x, y+q) \notin B] .
$$

Since $B$ is a Borel set, each of the sets $E$ and $F$ are Borel sets and therefore $C$ is $\Pi_{1}^{1}$. If each section of $B$ is countable, then for each $x \in X$, both $E_{x}$ and $F_{x}$ are countable, and therefore $C$ is a Borel set.

By a transversal for an equivalence relation $E$ on $X$ we mean a set $S \subseteq X$ which meets every $E$ class in exactly one point. By a Vitali set we mean a transversal for the equivalence relation $x \sim y \Leftrightarrow(x-y) \in \mathbb{Q}$ on the space $\mathbb{R}$.

Theorem 2.2. The following statements are equivalent:

(i) There is a Borel set in $X \times \mathbb{R}$ which uniquely represents all the translates of $\mathbb{Q}$.

(ii) There is a $\boldsymbol{\Sigma}_{2}^{1}$ Vitali set $V$.

(iii) There is a $K_{\sigma}$ subset of $X \times \mathbb{R}$ which uniquely represents the translates of $\mathbb{Q}$. 
Proof. First, suppose the Borel set $B$ uniquely represents the translates of $\mathbb{Q}$. Let $U$ be a $\Pi_{1}^{1}$ uniformization of $B$. Let

$$
V=\operatorname{proj}_{2}((C(B) \times \mathbb{R}) \cap U) .
$$

Then $V$ is a $\Sigma_{2}^{1}$ set and $V$ meets each translate of $\mathbb{Q}$ in exactly one point. So, $V$ is a transversal for $\mathbb{R} / \mathbb{Q}$.

Second, suppose $V$ is a $\boldsymbol{\Sigma}_{2}^{1}$ Vitali set. Let $C$ be a $\boldsymbol{\Pi}_{1}^{1}$ subset of $X$ and $f$ a continuous one-to-one map of $C$ onto $V$. Let $F=\overline{\operatorname{Gr}(f)}$. If $x \in C$, then $F_{x}=$ $\{f(x)\}$. Also, let $M$ be a closed subset of $X \times[0,1]$ such that if $x \in C$, then $M_{x}$ is a nonempty compact set lying in $\mathbb{Q}$ and if $x \notin C$, then $M_{x}$ is an uncountable compact set (cf. [6], [12], and 27.4 of [7]). Let

$$
H=M+{ }_{2} F=\{(x, y+z):(x, y) \in M,(x, z) \in F\} .
$$

Then $H$ is a closed subset of $X \times \mathbb{R}$. Let

$$
B=\bigcup_{q \in \mathbb{Q}}\{(x, y+q):(x, y) \in H\} .
$$

Then $B$ is an $F_{\sigma}$ subset of $X \times \mathbb{R}$, and the sections of $B$ over $C$ uniquely represent the translates of $\mathbb{Q}$. Also, the other sections of $B$ if nonempty are uncountable. If $X$ is compact, then $B$ is $K_{\sigma}$ and we are done. In the general case, $X$ contains a homeomorphic copy of the Cantor space $2^{\omega}$, and we let $B \subseteq 2^{\omega} \times \mathbb{R} \subseteq X \times \mathbb{R}$ be as constructed when the domain space is $2^{\omega}$. This clearly works.

Finally, the third statement trivially implies the first statement.

We note the following corollary of the preceding theorem.

Theorem 2.3. If $V=L$, then there is a Borel set which uniquely represents the translates of $\mathbb{Q}$. If every $\boldsymbol{\Sigma}_{2}^{1}$ set is measurable, then there is no such Borel set.

Also, part of the argument of Theorem 2.2 gives the following.

Theorem 2.4. There is no Borel set $B \subset X \times \mathbb{R}$ such that all sections of $B$ are countable and $B$ uniquely represents all the translates of $\mathbb{Q}$.

Proof. Suppose $B$ were such a set. Then the set $C(B)$ would be a Borel set. Also, since each section of $B$ is countable, $B$ would have a Borel uniformization $U$. Then $V=\operatorname{proj}_{2}((C(B) \times R) \cap U)$ would be a Borel transversal for $\mathbb{R} / \mathbb{Q}$.

\section{UNiQUe REPRESENTATION OF THE FAMILY OF COUNTABLE SETS}

For any uncountable Polish spaces $X$ and $Y$ it follows from remark 1.6 that there is a Borel set in $X \times Y$ with countable sections uniquely representing the non-empty countable subsets of $Y$ if and only if there is a Borel set in $X \times \mathbb{R}$ with countable sections uniquely representing the non-empty countable subsets of $\mathbb{R}$. But, since such a set would uniquely represent the translates of $\mathbb{Q}$, theorem 2.4 tells us there is no such Borel set in $X \times \mathbb{R}$. Thus we have the following theorem due to Becker and (according to [1]) independently Blackwell (cf. theorem 1 of [1]).

Theorem 3.1. There is no Borel set $B \subseteq X \times Y$ such that all sections of $B$ are countable and $B$ uniquely represents all non-empty countable subsets of $Y$.

We now give another different sort of proof. The argument is similar to one in $[5]$. 
Proof. From remark 1.6 we may assume that $X=Y=\omega^{\omega}$. Assume such a Borel set exists in $\omega^{\omega} \times \omega^{\omega}$ and let $w$ be a Borel code for it, so $B=B(w)$. Consider the statement

where

$$
\varphi:=\varphi_{0} \wedge \varphi_{1} \wedge \varphi_{2}
$$

$$
\begin{gathered}
\varphi_{0}:=\forall u, v\left(B_{u}=B_{v} \neq \emptyset \rightarrow u=v\right), \\
\varphi_{1}:=\forall u\left(B_{u} \text { is countable }\right),
\end{gathered}
$$

and

$$
\varphi_{2}:=\forall x \exists y\left[\left\{x_{n}\right\} \subseteq B_{y} \wedge \forall z \in \Delta_{1}^{1}(w, y)\left(z \in B_{y} \rightarrow \exists n\left(z=x_{n}\right)\right)\right] .
$$

Note that $\varphi$ is true in $V$, and $\varphi$ is $\Pi_{2}^{1}$ by the bounded quantification theorem (theorem 4D.3 of [13]).

Now consider Cohen forcing, understood as adding $\omega$ many mutually generic Cohen reals. Let $G$ be a generic set and $x=x_{G}$ code the sequence of Cohen reals. Let $\hat{B}=(B(w))^{V[G]}$. By absoluteness the statement $\varphi$ for $\hat{B}$ holds in $V[G]$. From $\varphi_{1}$ we get that (in $V[G]$ ) all sections of $\hat{B}$ are countable. From $\varphi_{2}$ and the fact that all countable $\Delta_{1}^{1}(w, y)$ sets contain only $\Delta_{1}^{1}(w, y)$ reals we get that $\hat{B}$ represents all non-empty countable sets, and then from $\varphi_{0}$ we get that $\hat{B}$ uniquely represents the non-empty countable sets.

Let $y$ be the unique real witnessing that $\varphi_{2}$ holds for the generic real $x$. By homogeneity of the forcing notion it can be seen that for any $n \in \omega$, either $\emptyset \Vdash \dot{y}(n)=0$ or $\emptyset \Vdash \dot{y}(n)=1$. [Suppose $p \Vdash \dot{y}(n)=0$ and $q \Vdash \dot{y}(n)=1$. Let $\pi$ be an automorphism of the forcing notion $\mathbb{P}=\prod_{\omega} \omega^{<\omega}$ such that $p$ is compatible with $\pi(q)$, and where $\pi$ is of the form $\pi(n, s)=(\sigma(n), s)$ for some permutation $\sigma$ of $\omega$. If $G$ is a generic extending $p$ and $\pi(q)$, then for $y_{1}$ the unique real such that $\hat{B}_{y_{1}}=C\left(x_{G}\right)$ we have $y_{1}(n)=0$, and for $y_{2}$ the unique real such that $\hat{B}_{y_{2}}=C\left(x_{\pi(G)}\right)$ we have $y_{2}(n)=1$. However $y_{1}=y_{2}$ since $C(G)=C(\pi(G))$.] In particular $y \in V$ and does not depend on the generic $G$. Now for two mutually $V$-generic Cohen reals $x^{\prime}, x^{\prime \prime} \in V[G]$ it follows that

$$
\left\{x_{n}^{\prime}\right\}=\hat{B}_{y}=\left\{x_{n}^{\prime \prime}\right\} \text {, }
$$

which is absurd.

The previous Cohen forcing argument can also be recast as a category argument. Namely, define $A(x, y) \leftrightarrow C(x)=B_{y}$. A computation as in $\varphi_{2}$ above shows that $A$ is Borel, and clearly $A$ is also the graph of a function $f$ (note that $f$ is a total function, that is, $\left.\operatorname{dom}(f)=\omega^{\omega}\right)$. We claim that for each $n$ there is an $m$ such that $A_{n, m}:=\{x: f(x)(n)=m\}$ is comeager in $\omega^{\omega}$. This implies that $f$ is constant on a comeager set, which is clearly impossible. If the claim fails, then since each $A_{n, m}$ has the Baire property, we have that for some $n$, some $m_{1} \neq m_{2}$, and some basic open sets $N_{p}, N_{q}$ in $\omega^{\omega}$ (determined by sequences $p, q \in \omega^{<\omega}$ ) that $A_{n, m_{1}}$ is comeager on $N_{p}$ and $A_{n, m_{2}}$ is comeager on $N_{q}$. In countably many steps we may now build $x, y \in \omega^{\omega}$ with $x$ extending $p, y$ extending $q, x \in A_{n, m_{1}}, y \in A_{n, m_{2}}$, and $C(x)=C(y)$. This is a contradiction as we must have $f(x)=f(y)$ and yet $f(x)(n)=m_{1}, f(y)(n)=m_{2}$.

A simpler version of the above argument gives the next result, which is that there is no analytic set uniquely representing all non-empty countable sets, assuming either every $\boldsymbol{\Sigma}_{2}^{1}$ set has the Baire property or $\boldsymbol{\Sigma}_{3}^{1}$ absoluteness holds between $V$ 
and the Cohen extensions $V[G]$. In the first case, this strengthens theorem 2 of [1] (which says, in our terminology, that there is no analytic set which is uniquely universal for the countable sets; however, Becker's proof also shows the stronger result).

Theorem 3.2. Assume either (1) every $\boldsymbol{\Sigma}_{2}^{1}$ set has the Baire property or (2) $\boldsymbol{\Sigma}_{3}^{1}$ absoluteness holds between $V$ and the Cohen extensions $V[G]$. Then there is no analytic set $B \subseteq \omega^{\omega} \times \omega^{\omega}$ which uniquely represents the non-empty countable sets.

Proof. Suppose first that the absoluteness hypothesis holds. Instead of considering $\varphi$ we consider

where

$$
\psi:=\psi_{0} \wedge \psi_{1}
$$

$$
\psi_{0}:=\forall u, v\left[\exists x\left(B_{u}=B_{v}=C(x)\right) \rightarrow u=v\right]
$$

and

$$
\psi_{1}:=\forall x \exists y\left(C(x)=B_{y}\right) .
$$

Note that $\psi_{0}$ is $\boldsymbol{\Pi}_{2}^{1}$ and $\psi_{1}$ is $\boldsymbol{\Pi}_{3}^{1}$, so under our hypothesis they are all absolute between $V$ and $V[G]$, where $G$ is generic for adding $\omega$ many Cohen reals (equivalently, one Cohen real). The same forcing argument as before yields a contradiction.

If the Baire property hypothesis holds, we proceed as in the category argument above except now the relation $A$ giving the graph of $f$ is the conjunction of a $\boldsymbol{\Sigma}_{1}^{1}$ and a $\boldsymbol{\Pi}_{1}^{1}$ set. By hypothesis such functions are Baire measurable, and the argument finishes as before.

Thus in particular if $\mathrm{MA}+\neg \mathrm{CH}$ holds or if $\mathbf{\Pi}_{1}^{1}$-determinacy holds, there is no chance of finding unique representations for countable sets, let alone for $K_{\sigma}$ sets or $F_{\sigma}$ sets.

Corollary 3.3. Let $x$ be Cohen generic over $L$. Then in $L[x]$ there is a $\boldsymbol{\Sigma}_{1}^{1}$ set which is uniquely universal for the countable sets, but there is no (lightface) $\Sigma_{1}^{1}$ set which even uniquely represents the countable sets.

Proof. Theorem 3 of [1], relativized to $x$ shows that in $L[x]$ there is a $\boldsymbol{\Sigma}_{1}^{1}$ set with countable sections which uniquely represents the countable sets. On the other hand, (lightface) $\Sigma_{3}^{1}$ absoluteness holds between $L[x]$ and $L[x][y]$ where $y$ is Cohen generic over $L[x]$. [If the $\Sigma_{3}^{1}$ statement $\phi$ holds in $L[x][y]$, then for some $(p, q) \in \mathbb{P} \times \mathbb{P}$, $(p, q) \Vdash_{\mathbb{P} \times \mathbb{P}} \phi$ where $\mathbb{P}=\left(\omega^{<\omega}, \leq\right)$ is the Cohen partial order. By homogeneity, $\emptyset \Vdash_{\mathbb{P} \times \mathbb{P}} \phi$. Since $\mathbb{P} \cong \mathbb{P} \times \mathbb{P}, \emptyset \Vdash_{\mathbb{P}} \phi$, and so $\phi$ holds in $L[x]$.] Suppose in $L[x]$ that $B \subseteq \omega^{\omega} \times \omega^{\omega}$ were $\Sigma_{1}^{1}$ and uniquely represented the countable sets. Then the statement $\psi$ from the previous proof would be (lightface) $\Pi_{3}^{1}$, and so absolute between $L[x]$ and $L[x][y]$. The same proof as before then yields a contradiction.

We can also state an analog of theorem 2.2 for the class of countable sets using the equivalence relation $E_{c}$ on $\omega^{\omega}$ defined by $x E_{c} y$ iff $C(x)=C(y)$.

Theorem 3.4. The following are equivalent.

(1) There is a Borel set $B \subseteq \omega^{\omega} \times \omega^{\omega}$ which uniquely represents the non-empty countable subsets of $\omega^{\omega}$.

(2) There is a $\boldsymbol{\Sigma}_{2}^{1}$ transversal for the equivalence relation $E_{c}$.

(3) There is an $F_{\sigma}$ set $B \subseteq \omega^{\omega} \times \omega^{\omega}$ which uniquely represents the countable subsets of $\omega^{\omega}$. 
Proof. Suppose $B \subseteq \omega^{\omega} \times \omega^{\omega}$ uniquely represents the non-empty countable subsets of $\omega^{\omega}$. Define $R(x, y) \leftrightarrow\left(B_{x}=C(y)\right)$. Easily $R \in \boldsymbol{\Pi}_{1}^{1}$. Let $R^{\prime} \in \boldsymbol{\Pi}_{1}^{1}$ uniformize $R$. Define $S \subseteq \omega^{\omega}$ by

$$
S(y) \leftrightarrow \exists x R^{\prime}(x, y) .
$$

$S \in \Sigma_{2}^{1}$ and easily is a transversal for $E_{c}$.

Suppose now $S \subseteq \omega^{\omega}$ is a $\Sigma_{2}^{1}$ transversal for $E_{c}$. Let $A \subseteq \omega^{\omega}$ be $\Pi_{1}^{1}$ and $f: A \rightarrow S$ a continuous one-to-one map from $A$ onto $S$. In fact, $f$ can be taken to be the decoding map $f(x)=(x)_{0}$, so $f$ is the restriction to $A$ of a continuous function $f^{\prime}: \omega^{\omega} \rightarrow \omega^{\omega}$. Let $M \subseteq \omega^{\omega} \times \omega^{\omega}$ be closed such that if $x \in A$, then $M_{x}=\emptyset$ and if $x \notin A$, then $M_{x}$ is uncountable. Define

$$
B(x, y) \leftrightarrow M(x, y) \vee \exists n\left[\left(y=\left(f^{\prime}(x)\right)_{n}\right)\right] .
$$

$B$ is clearly $F_{\sigma}$. If $x \notin A$, then $B_{x}$ is uncountable, and $\left\{B_{x} \mid x \in A\right\}$ uniquely represents the non-empty countable sets since $f$ is one-to-one and $f^{\prime \prime} A=S$, which is a transversal for $E_{c}$. It is trivial to now modify $B$ so that the empty set is also uniquely represented.

The third statement trivially implies the first.

Problem 3.5. Is the existence of a Borel subset of $\mathbb{R} \times \mathbb{R}$ uniquely representing the translates of $\mathbb{Q}$ equivalent to the existence of a Borel subset of $\mathbb{R} \times \mathbb{R}$ uniquely representing the non-empty countable subsets of $\mathbb{R}$ ?

We guess the answer is no. One reason for feeling this is that the Vitali equivalence relation (which is bireducible to the equivalence relation $E_{0}$ of eventual equality on $\omega^{\omega}$ ) is the minimal "non-smooth" countable Borel equivalence relation (i.e., not having a Borel selector), whereas every countable Borel equivalence relation embeds into $E_{c}$. We refer the reader to [4] for further details and precise statements of these results.

As a corollary to theorem 3.4 we have that if $V=L$, then there is an $F_{\sigma}$ set which uniquely represents the countable sets. In particular, $V=L$ implies $U\left(\boldsymbol{\Delta}_{1}^{1}, \boldsymbol{\Sigma}_{2}^{0}, \mathcal{C}_{\text {ctbl }}\right)$.

Theorem 3.6. Assume $V=L$. Then there is an $F_{\sigma}$ set $B \subseteq \omega^{\omega} \times \omega^{\omega}$ such that all countable sets are uniquely represented by $B$.

Proof. Let $<$ be a $\Delta_{2}^{1}$-good wellordering of $\omega^{\omega}$ (see section 5A of [13]). Let

$$
R(x) \Leftrightarrow \forall y<x(C(y) \neq C(x)) .
$$

Then $R$ is a $\Sigma_{2}^{1}$ selector for $E_{c}$.

A similar argument can show that from $V=L$ there exists a Borel set with $F_{\sigma}$ sections and uniquely representing all $K_{\sigma}$ sets, that is, $U\left(\boldsymbol{\Delta}_{1}^{1}, \boldsymbol{\Sigma}_{2}^{0}, K_{\sigma}\right)$. To consider this problem first recall a standard coding of $K_{\sigma}$ subsets of $\omega^{\omega}$. To start with, all non-empty compact subsets of $\omega^{\omega}$ can be coded by non-empty, pruned, finite splitting trees. These trees form a Borel subset of $\mathcal{P}\left(\omega^{<\omega}\right)$ and thus form a standard Borel space. It follows that there is a Borel one-to-one correspondence $x \mapsto T_{x}$ from $\omega^{\omega}$ onto the space of all non-empty, pruned, finite-splitting trees on $\omega$. Note that the map $x \mapsto\left[T_{x}\right]$ is a Borel one-to-one correspondence from $\omega^{\omega}$ onto the space of all non-empty compact subsets of $\omega^{\omega}$. Now to each $x \in \omega^{\omega}$ we associate a non-empty $K_{\sigma}$ set

$$
K(x):=\bigcup_{n \in \omega}\left[T_{x_{n}}\right]
$$


Theorem 3.7. Assume $V=L$. Then there is a Borel set $B \subseteq \omega^{\omega} \times \omega^{\omega}$ such that all sections of $B$ are $F_{\sigma}$ and all non-empty $K_{\sigma}$ sets are uniquely represented by $B$.

Proof. Again let $<$ be a good $\Delta_{2}^{1}$ wellordering of the reals. Let

$$
R(x) \Leftrightarrow \forall y<x(K(y) \neq K(x)) .
$$

Then $R$ is $\Sigma_{2}^{1}$. Using $\Pi_{1}^{1}$ uniformization one can find $P \subseteq \omega^{\omega}$ which is $\Pi_{1}^{1}$ such that

$$
R(x) \Leftrightarrow \exists w P(\langle x, w\rangle) \Leftrightarrow \exists ! w P(\langle x, w\rangle) .
$$

Let $S$ be the complement of $P$ and let $F \subseteq \omega^{\omega} \times \omega^{\omega}$ be closed such that

$$
S(u) \Leftrightarrow \exists v F(u, v) .
$$

Consider $F^{\prime} \subseteq \omega^{\omega} \times \omega^{\omega}$ defined by

$$
F^{\prime}(u, v) \Leftrightarrow F\left(u,(v)_{0}\right) .
$$

It is easy to see that $F^{\prime}$ is closed and

$$
S(u) \Leftrightarrow \exists v F^{\prime}(u, v) .
$$

Moreover, if $S(u)$ holds, then the set $\left\{v \mid F^{\prime}(u, v)\right\}$ contains a homeomorphic copy of $\omega^{\omega}$ as a closed subset; hence it is not $K_{\sigma}$.

We now define the set $B$ by

$$
B(u, v) \Leftrightarrow F^{\prime}(u, v) \text { or letting } u=\langle x, w\rangle, v \in\left[T_{x_{n}}\right] \text { for some } n \in \omega .
$$

Then $B$ is obviously Borel and has $F_{\sigma}$ sections. If $x$ is the <-least real coding $K(x)$ and $w$ is the unique witness for $P(\langle x, w\rangle)$, then $B_{\langle x, w\rangle}=K(x)$. Otherwise, if $P(\langle x, w\rangle)$ fails, then $F_{\langle x, w\rangle}^{\prime}$ is non-empty and therefore not $K_{\sigma}$ by our construction; thus $B_{\langle x, w\rangle}$ is not $K_{\sigma}$.

In view of the above theorems one can ask the following question:

Question 3.8. Does there exist a Borel set $B \subseteq \omega^{\omega} \times \omega^{\omega}$ with all sections $K_{\sigma}$ and uniquely representing all countable sets?

We will answer this question in the next section.

\section{Representation of SCATtered Sets And $K_{\sigma}$ Sets}

Many descriptive set theoretic results about countable sets can be generalized to $K_{\sigma}$ 's. It is a common rule of thumb that $K_{\sigma}$ sets behave more like countable sets than general $F_{\sigma}$ sets. One might guess that the answer to question 3.8 is no in ZFC based on this rule of thumb. However, it is our main result here to show that the answer is consistently yes.

Theorem 4.1. Assume $V=L$. Then there is a Borel set $B \subseteq X \times Y$ with all sections $K_{\sigma}$ which uniquely represents the countable subsets of $Y$.

In other words, we show that $U\left(\boldsymbol{\Delta}_{1}^{1}, K_{\sigma}, \mathcal{C}_{\text {ctbl }}\right)$ is consistent. Note that by remark 1.6 we may assume that $X=Y=\omega^{\omega}$. The rest of this section is devoted to proving theorem 4.1. We begin with some results about small countable sets, the scattered sets.

Definition 4.2. A countable subset $C$ of a Polish space is large if there is a nonempty $C^{*} \subseteq C$ such that $C^{*}$ is dense in itself. Otherwise $C$ is scattered. 
Scattered sets can be characterized by considering the classical Cantor-Bendixson derivatives. We recall its definition below. Let $C$ be a subset of a Polish space. Then the (Cantor-Bendixson) derivative of $C$ is the set $C^{\prime}$ of all accumulation points of $C$ that are in $C$. For $\alpha<\omega_{1}$, the $\alpha$-th (Cantor-Bendixson) derivative of $C, C_{\alpha}$, is obtained by iterating the derivative operation $\alpha$ times, i.e.,

$$
\begin{aligned}
C_{0} & =C, \\
C_{\alpha+1} & =\left(C_{\alpha}\right)^{\prime}, \\
C_{\lambda} & =\bigcap_{\alpha<\lambda} C_{\alpha}, \text { for limit } \lambda .
\end{aligned}
$$

The (Cantor-Bendixson) rank of $C$ is the least $\alpha$ such that $C_{\alpha}=C_{\alpha+1}$. Of course, the rank of $C$ is a countable ordinal. We recall the following classical result (cf. theorem 4 of $\S 6$ of [9]).

Proposition 4.3. Let $X$ be a Polish space. The following are equivalent for $C \subseteq X$ :

(i) $C$ is scattered;

(ii) for some $\alpha<\omega_{1}, C_{\alpha}=\emptyset$;

(iii) $C$ is a countable $G_{\delta}$.

It is worth noting that there is no analytic set which is universal for all scattered sets in an uncountable Polish space $Y$. In fact, no analytic set can be universal for any family of scattered sets with unbounded Cantor-Bendixson index.

Theorem 4.4 ([8], [11], [3]). Let $X$ and $Y$ be Polish spaces. If $A \subseteq X \times Y$ is analytic and all sections of $A$ are scattered, then there is a countable ordinal $\alpha$ such that the Cantor-Bendixson orders of all sections of $A$ are bounded by $\alpha$.

The argument given in [3] actually holds for a general class of inductive operators. In view of proposition 4.3 we may rephrase theorem 4.4 as saying there is no analytic set which is universal for the class of countable $\boldsymbol{\Delta}_{2}^{0}$ sets. We will show in Section 5 that there is no analytic set universal for the $\boldsymbol{\Delta}_{2}^{0}$ sets.

In contrast it is provable in ZFC that the scattered sets or small countable sets can be uniquely represented. In the following theorem we show a stronger result.

Theorem 4.5. There is a Borel set $B \subseteq \omega^{\omega} \times \omega^{\omega}$ such that

(a) all sections of $B$ are $K_{\sigma}$,

(b) all non-empty scattered sets are uniquely represented, and

(c) every section of $B$ is either scattered or else uncountable.

Proof. First fix an enumeration $\left\{N_{i}\right\}_{i \in \omega}$ of all basic open sets of $\omega^{\omega}$.

For each $w \in 2^{\omega}$ define a binary relation $<_{w}$ on a subset of $\omega$ by

$$
m<_{w} n \Leftrightarrow w(\langle m, n\rangle)=1 .
$$

The domain of $<_{w}$ is the set

$$
\operatorname{dom}\left(<_{w}\right)=\{m \in \omega \mid \exists n(w(\langle m, n\rangle)=1 \text { or } w(\langle n, m\rangle)=1)\} .
$$

Let $L$ be the set of all $w \in 2^{\omega}$ such that $<_{w}$ is a linear order with a least element and that each $n \in \operatorname{dom}\left(<_{w}\right)$ has an immediate successor unless it is $<_{w}$-largest. If $w \in L$ and $n \in \operatorname{dom}\left(<_{w}\right)$ we denote by $n_{w}^{+}$the immediate successor of $n$ in $<_{w}$ if $n$ is not $<_{w}$-largest. If there is no danger of confusion we will omit the subscript and simply write $n^{+}$. We also write

$$
m \leq_{w} n \Leftrightarrow m<_{w} n \text { or } m=n .
$$


Definition 4.6. We call a real $z \in \omega^{\omega}$ adequate if $z=\langle w, k, x\rangle$, where $w \in 2^{\omega}$, $k \in \omega, x \in \omega^{\omega}$, and the following conditions are satisfied:

(a) $w \in L$;

(b) if $n \notin \operatorname{dom}\left(<_{w}\right)$, then $x_{n}=0$ (the constant 0 element of $\omega^{\omega}$ );

(c) if there is no $<_{w}$-largest element, then $k=0$;

(d) if $n \in \operatorname{dom}\left(<_{w}\right)$ is $<_{w}$-largest and $k>0$, then $\left(x_{n}\right)_{m}=0$ for all $m \geq k$;

(e) letting, for $w \in L, k \in \omega$ and $n \in \operatorname{dom}\left(<_{w}\right)$,

$S_{n}^{z}=\left\{\left(x_{p}\right)_{m} \mid n \leq_{w} p\right.$ and if $p$ is $<_{w}$-largest and $k>0$, then $\left.m<k\right\}$

and

$D_{n}^{z}=S_{n}^{z} \backslash S_{n^{+}}^{z}$, if $n$ is not largest, and $S_{n}^{z}$ otherwise,

we have that:

(e1) for each $n \in \operatorname{dom}\left(<_{w}\right), D_{n}^{z}$ is the set of all isolated points of $S_{n}^{z}$;

(e2) if $\left(x_{n}\right)_{m},\left(x_{n}\right)_{l} \in D_{n}^{z}$ and $m<l$, then $i_{m}<i_{l}$, where $i_{m}$ and $i_{l}$ are respectively the smallest indices such that

$$
N_{i_{m}} \cap S_{n}^{z}=\left\{\left(x_{n}\right)_{m}\right\} \text { and } N_{i_{l}} \cap S_{n}^{z}=\left\{\left(x_{n}\right)_{l}\right\} ;
$$

(e3) for each $n \in \operatorname{dom}\left(<_{w}\right)$ the smallest index $i$ such that $\left\{\left(x_{n}\right)_{0}\right\}=N_{i} \cap S_{n}^{z}$ is equal to $n$.

(e4) for $n<_{w} m$ in $\operatorname{dom}\left(<_{w}\right)$, every real in $S_{m}^{z}$ is a limit point of $D_{n}^{z}$.

If $z=\langle w, k, x\rangle$ is adequate let $S_{z}=S_{n}^{z}$ where $n$ is the $<_{w}$-least element.

Let $A$ be the set of all adequate reals.

Remark 4.7. For adequate $z=\langle w, k, x\rangle, x$ attempts to code the Cantor-Bendixson derivation sequence of the set $S_{z}$ of reals coded by $x$. For $n \in \operatorname{dom}\left(<_{w}\right), S_{n}^{z}$ is a candidate for the $\alpha^{\text {th }}$ Cantor-Bendixson derivative, where $\alpha$ is the rank of $n$ in $<_{w}$, and $D_{n}^{z}$ is a candidate for the reals removed at stage $\alpha . w$ attempts to code the wellorder which is canonically determined from this sequence. (e2) says that the reals in $D_{n}^{z}$ are coded by $x_{n}$ in the order corresponding to the basic open sets that isolate them in $S_{n}^{z}$. (e3) says that the order $<_{w}$ is the one canonically obtained from this derivation. (e4) says that every point in $S_{m}^{z}$ is a limit point of the set $D_{n}^{z}$ for $n<_{w} m$ (not just a limit of $S_{n}^{z}$ ).

Lemma 4.8. A is Borel.

Proof. By straightforward computations one can verify that each condition in the definition of adequacy is Borel.

Lemma 4.9. If $C$ is scattered, then there is a unique adequate real $z$ such that $C=S_{z}$. Furthermore if $z=\langle w, k, x\rangle$, then $<_{w}$ is a wellorder.

Proof. Let $C$ be small or scattered, $\alpha$ be its Cantor-Bendixson rank and $\left\{C_{\beta}\right\}_{\beta<\alpha}$ be the iterated Cantor-Bendixson derivatives of $C$. For each $\beta<\alpha$ also let $D_{\beta}=$ $C_{\beta} \backslash C_{\beta+1}$. Then $D_{\beta}$ is the set of all isolated points of $C_{\beta}$. For each element $y \in D_{\beta}$ let $i_{y}$ be the smallest index such that $N_{i_{y}} \cap C_{\beta}=\{y\}$. Note that $y \mapsto i_{y}$ is a one-to-one function from $C$ into $\omega$. [Suppose $y_{1} \neq y_{2}$ are in $C$ and $i_{y_{1}}=i_{y_{2}}=i$. Say $y_{1} \in D_{\beta_{1}}, y_{2} \in D_{\beta_{2}}$ with $\beta_{1} \leq \beta_{2}$. Then $\left\{y_{1}\right\}=N_{i} \cap C_{\beta_{1}} \supseteq N_{i} \cap C_{\beta_{2}}=\left\{y_{2}\right\}$, a contradiction.] Thus $D_{\beta}$ can be canonically enumerated according to the natural order of the set $\left\{i_{y} \mid y \in D_{\beta}\right\}$. When $\beta+1<\alpha$ the set $D_{\beta}$ is necessarily infinite; in this case we denote the canonical enumeration of $D_{\beta}$ by $\left\{x_{m}^{\beta}\right\}_{m<\omega}$. In case $\alpha$ 
is a successor ordinal and $\beta+1=\alpha$ the set $D_{\beta}$ might be finite but is certainly nonempty. Thus the canonical enumeration of $D_{\beta}$ takes the form of either $\left\{x_{m}^{\beta}\right\}_{m<\omega}$ or $\left\{x_{m}^{\beta}\right\}_{m<k}$ for some $k>0$.

We can now define an adequate real $z$ coding the set $C$. Let $\operatorname{dom}\left(<_{w}\right)$ be the set of all $i_{x_{0}^{\beta}}$ for $\beta<\alpha$, and define $<_{w}$ by

$$
i_{x_{0}^{\beta}}<{ }_{w} i_{x_{0}^{\gamma}} \Leftrightarrow \beta<\gamma .
$$

Then $<_{w}$ is a wellorder with order type $\alpha$. This defines the first component of $z$. If $\alpha=\beta+1$ and $D_{\beta}$ is finite, define $k$ to be the cardinality of $D_{\beta}$. Otherwise let $k=0$. Finally the correct definition of $x$ is obvious from the descriptions in the preceding paragraph and from the requirements of (b) and (d) in the definition of adequacy. Let $z=\langle w, k, x\rangle$. Since $C$ is scattered, it is easy to check that for $\beta<\gamma<\alpha$, every real in $C_{\gamma}$ is a limit point of $D_{\beta}$, and so (e4) will be satisfied. It is easy to see that $C=S_{z}$.

To see uniqueness, suppose that $z^{\prime}=\left\langle w^{\prime}, k^{\prime}, x^{\prime}\right\rangle$ is adequate and $S_{z^{\prime}}=C$. We first show that $<_{w^{\prime}}$ is a wellordering. Suppose not, and let $n_{0}>_{w^{\prime}} n_{1}>_{w^{\prime}} \ldots$ be an infinite decreasing chain. We claim that any real of the form $\left(x_{n_{i}}\right)_{m}$ lies in $C_{\alpha}$ for all countable ordinals $\alpha$, a contradiction to $C$ being scattered. Suppose this claim holds for $\alpha$. Since $n_{i}>{ }_{w^{\prime}} n_{i+1}$, (e4) gives that every real of the form $\left(x_{n_{i}}\right)_{m}$ is a limit of reals in $C_{\alpha}$, and thus in $C_{\alpha+1}$. Limit stages are trivial, and this shows the claim. So, $<_{w^{\prime}}$ is a wellordering. Using (e1) it is straightforward to prove by induction on $\beta=|n|_{<_{w^{\prime}}}$, for $n \in \operatorname{dom}\left(<_{w^{\prime}}\right)$, that $\left\{\left(x_{n}^{\prime}\right)_{m}\right\}_{m \in \omega}=D_{\beta}$. The definition of adequate, in particular (e2) and (e3), now shows that $w^{\prime}, k^{\prime}$, and $x^{\prime}$ can be recovered from the derivation sequence $\left\{D_{\beta}\right\}_{\beta<\alpha}$, and thus $z^{\prime}=z$.

Lemma 4.10. If $z=\langle w, k, x\rangle$ is adequate and $<_{w}$ is a wellorder, then $S_{z}$ is scattered.

Proof. If $<_{w}$ is a wellorder and $z=\langle w, k, x\rangle$ is adequate, then as in the last paragraph of the proof of the previous lemma, $z$ codes the iterated Cantor-Bendixson derivatives of the set $S_{z}$ which terminates with the empty set. Therefore $S_{z}$ is small.

Lemma 4.11. If $z=\langle w, k, x\rangle$ is adequate and $S_{z}$ is not small, then $<_{w}$ is illfounded.

Proof. The proof is immediate from the previous lemma.

We will also need the following lemma on uniform Borel cofinalities of elements in $<_{w}$.

Lemma 4.12. There is a Borel function

$$
\operatorname{Cof}:\left\{(w, n, j) \in L \times \omega^{2} \mid n \in \operatorname{dom}\left(<_{w}\right)\right\} \rightarrow \omega
$$

such that

(a) for $w \in L, n \in \operatorname{dom}\left(<_{w}\right)$ and $j \in \omega, \operatorname{Cof}(w, n, j) \in \operatorname{dom}\left(<_{w}\right)$ and $\operatorname{Cof}(w, n, j)$ $<_{w} n$ unless $n$ is the $<_{w}$-least element;

(b) if $w \in L$ and $n \in \operatorname{dom}\left(<_{w}\right)$ has an immediate predecessor in $<_{w}$, then $\operatorname{Cof}(w, n, j)_{w}^{+}=n$ for all $j \in \omega$; 
(c) if $w \in L, n \in \operatorname{dom}\left(<_{w}\right)$ is not $<_{w}$-least and $n$ does not have an immediate predecessor in $<_{w}$, then

(c1) if $j<j^{\prime}$, then $\operatorname{Cof}(w, n, j)<{ }_{w} \operatorname{Cof}\left(w, n, j^{\prime}\right)$, and

(c2) for any $q \in \operatorname{dom}\left(<_{w}\right)$ with $q<_{w} n$ there is a $j$ such that $q<_{w}$ $\operatorname{Cof}(w, n, j)$.

Proof. Clause (b) gives the definition of Cof in a case characterized by a Borel condition. Thus we focus on (c) and assume the hypotheses hold. We define Cof by induction on $j$. Let $\operatorname{Cof}(w, n, 0)$ be the smallest element $p_{0} \in \operatorname{dom}\left(<_{w}\right)$ such that $p_{0}<_{w} n$. Such a $p_{0}$ exists since $n$ is not $<_{w}$-least. In general suppose $p_{j}=\operatorname{Cof}(w, n, j)$ has been defined for $j \in \omega$. Define $p_{j+1}=\operatorname{Cof}(w, n, j+1)$ to be the smallest element of $\operatorname{dom}\left(<_{w}\right)$ such that $p_{j}<_{w} p_{j+1}$ and $p_{j+1}<_{w} n$. Such a $p_{j+1}$ exists since $n$ does not have an immediate predecessor in $<_{w}$. This finishes our definition of the function Cof.

Apparently the function is Borel and satisfies clauses (a), (b) and (c1). To see that (c2) holds, let $q<_{w} n$. Note that the sequence $\left\{p_{j}\right\}_{j \in \omega}$ is strictly increasing in the usual order of natural numbers. Let $i$ be maximal such that $p_{i}<q$. If $p_{i} \geq_{w} q$, we are done. Otherwise, by construction $p_{i+1}=q$, so in either case $p_{i+1} \geq_{w} q$.

We next define a Borel set $B_{0} \subseteq A \times \omega^{\omega}$ with $K_{\sigma}$ sections. For each $n, m \in \omega$ we define a Borel set $B_{0}^{n, m} \subseteq A \times \omega^{\omega}$ with compact sections, and then we set $B_{0}=\bigcup_{n, m} B_{0}^{n, m}$. Fix $z \in A$, say $z=\langle w, k, x\rangle$, and fix $n, m \in \omega$. We inductively define for $s \in 2^{<\omega}$ sequences $t_{s} \in \omega^{<\omega}$ with the following properties:

(1) if $s_{1} \subset s_{2}$, then $t_{1} \subset t_{2}$;

(2) for any $s$, if $t_{s^{\wedge} 0}$ and $t_{s^{\prime} 1}$ are incompatible, then $t_{s}=t_{s^{\wedge} 0} \cap t_{s^{\wedge} 1}$;

(3) for any $s$, if $t_{s^{\wedge} 0}$ and $t_{s^{\wedge} 1}$ are compatible, then the set $\left\{t_{s^{\prime}} \mid s \subset s^{\prime}\right\}$ is linearly ordered by $\subset$.

The $t_{s}$ will thus define a finite splitting subtree $T_{z}$ of $\omega^{<\omega}$ (namely, $t \in T_{z}$ iff $t$ is an initial segment of some $\left.t_{s}\right)$, and $\left(B_{0}^{n, m}\right)_{z}$ will be the compact set of branches $\left[T_{z}\right] \subseteq \omega^{\omega}$ through this tree. As we define the $t_{s}$ we will also define reals $u_{s} \in S_{z}$ and integers $n_{s}$. We will have that $t_{s}$ is an initial segment of $u_{s}$, and $u_{s \frown 0^{j}}=u_{s}$ for all $j$, where $0^{j}$ is the 0 sequence of length $j$.

Roughly speaking, $T_{z}$ will be the tree of attempts to find an infinite decreasing chain in $<_{w}$ starting from $n$. The tree $T_{z}$ will have the property that if $n$ is in the illfounded part of $<_{w}$, then $T_{z}$ will define an uncountable set, and if $n$ is in the wellfounded part of $<_{w}$, then $\left[T_{z}\right] \subseteq S_{z}$.

To begin, if $n \notin \operatorname{dom}\left(<_{w}\right)$ or $n$ is maximal in $<_{w}$ and $m \geq k$, then we stop the construction and set $\left(B_{0}^{n, m}\right)_{z}=\emptyset$. Otherwise, let $u_{\emptyset}=\left(x_{n}\right)_{m}, t_{\emptyset}=\emptyset$, and $n_{\emptyset}=n$. For every $j$, let $u_{0^{j}}=u_{\emptyset}$.

If $n_{\emptyset}$ is $<_{w}$-least, then make arbitrary definitions of $\left\{t_{s}\right\}_{s \in 2<\omega}$ to maintain that each $t_{s} \subseteq u_{\emptyset}$. Otherwise, consider $n^{\prime}=\operatorname{Cof}\left(w, n_{\emptyset}, 0\right)$. By our construction, $n^{\prime}<_{w}$ $n_{\emptyset}$. From $(\mathrm{e} 4), u_{\emptyset}$ is an accumulation point of the set $D_{n^{\prime}}^{z}$. Hence there exists a smallest $m^{\prime}$ such that $\left(x_{n^{\prime}}\right)_{m^{\prime}}$ has a nonempty intersection with $u_{\emptyset}$. Recall $u_{\langle 0\rangle}=u_{\emptyset}$ and let $u_{\langle 1\rangle}=\left(x_{n^{\prime}}\right)_{m^{\prime}}$. Let $l_{\emptyset}>0$ be the largest such that $u_{\langle 0\rangle} \uparrow l_{\emptyset}=u_{\langle 1\rangle} \uparrow l_{\emptyset}$. Define $t_{\langle 0\rangle}=u_{\langle 0\rangle} \uparrow\left(l_{\emptyset}+1\right)$ and $t_{\langle 1\rangle}=u_{\langle 1\rangle} \uparrow\left(l_{\emptyset}+1\right)$. Let $n_{\langle 0\rangle}=n, n_{\langle 1\rangle}=n^{\prime}$. For any finite sequence $\overrightarrow{0}$ consisting of all 0 's, let $u_{\langle 1\rangle-\overrightarrow{0}}=u_{\langle 1\rangle}$.

Proceeding by induction on $j$, suppose we have defined $t_{0^{j}{ }_{0}} \subseteq u_{0^{j}{ }_{0}}=u_{\emptyset}$,

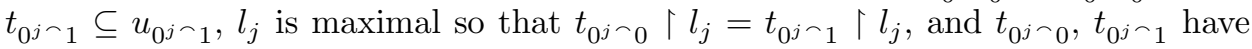


length $l_{j}+1$. We now make the inductive definitions. Consider $n^{\prime}=\operatorname{Cof}\left(w, n_{\emptyset}, j\right)$ and note that $n^{\prime}<_{w} n_{\emptyset}$ and that $u_{\emptyset}$ is an accumulation point of $D_{n^{\prime}}^{z}$. Thus there is a smallest $m^{\prime}$ such that $t_{0^{j}-0} \subseteq\left(x_{n^{\prime}}\right)_{m^{\prime}}$, and hence the intersection of $\left(x_{n^{\prime}}\right)_{m^{\prime}}$ with $u_{\emptyset}$ has length $>l_{j}$. Let $l_{j+1}>l_{j}$ be the largest such that $\left(x_{n^{\prime}}\right)_{m^{\prime}} \uparrow l_{j+1}=u_{\emptyset} \uparrow l_{j+1}$. Let $u_{0^{j+1} \uparrow_{1}}=\left(x_{n^{\prime}}\right)_{m^{\prime}}$. We have already defined $u_{0^{j+1} \frown_{0}}=u_{\emptyset}$. Let $t_{0^{j+1} \frown_{0}}=u_{\emptyset} \uparrow$ $\left(l_{j+1}+1\right)$ and $t_{0^{j+1} \imath_{1}}=u_{0^{j+1} \frown 1} \uparrow\left(l_{j+1}+1\right)$. Let $n_{\left\langle 0^{j+1} \frown 0\right\rangle}=n, n_{\left\langle 0^{j+1} \frown 1\right\rangle}=n^{\prime}$.

For the general inductive definition of $\left\{t_{s}\right\}_{s \in 2<\omega}$, let $s$ be a sequence ending with a 1 and suppose $t_{s} \subseteq u_{s} \in S_{z}$ are already defined. If $u_{s}=\left(x_{n_{s}}\right)_{m_{s}}$ and $n_{s}$ is $<_{w^{-}}$ least, then make arbitrary definitions of $\left\{t_{s^{\prime}}\right\}_{s \subset s^{\prime}}$ to maintain that each $t_{s^{\prime}} \subseteq u_{s}$. Otherwise, for every $j$ we define $t_{s^{\frown} 0^{j} \frown 0}, t_{s^{\frown} 0^{j} \frown 1}$, and $u_{s^{\frown} 0^{j}-1}$ as above but starting with $t_{s}$ instead of $t_{\emptyset}$, and using $n_{s}$ in place of $n$.

This finishes the definition of the sequence $\left\{t_{s}\right\}_{s \in 2}<\omega$. The construction obviously guarantees clauses (1)-(3) of the desired properties.

Lemma 4.13. If $C$ is scattered, then for the unique $z \in A$ with $C=S_{z}$ we have that $\left(B_{0}\right)_{z} \subseteq C$.

Proof. Let $z=\langle w, k, x\rangle$. From the construction of $B_{0}$ it is clear that every $y \in\left(B_{0}\right)_{z}$ corresponds to a descending sequence in the order $<_{w}$. Namely, if $y$ is the limit of $t_{s}$ for $s$ an initial segment of $u \in 2^{\omega}$, then for every $n$ such that $u(n)=1$ we have that $n_{u \uparrow(n+1)}<_{w} n_{u \uparrow n}$ and if $u(n)=0$, then $n_{u \uparrow(n+1)}=n_{u \uparrow n}$. Now if $C$ is small, then $<_{w}$ is a wellorder and every descending sequence in $<_{w}$ is finite, and thus $u$ is eventually equal to 0 . It follows that each $y \in\left(B_{0}\right)_{z}$ is an element of $S_{z}$; thus $\left(B_{0}\right)_{z} \subseteq C=S_{z}$.

Lemma 4.14. If $z=\langle w, k, x\rangle \in A$ and $<_{w}$ is illfounded, then $\left(B_{0}\right)_{z}$ is uncountable.

Proof. Start with an $n, m$, with $n$ in the illfounded part of $<_{w}$. By cofinality $\operatorname{Cof}(w, n, j)$ is in the illfounded part of $<_{w}$ for some $j>0$. By our construction of $B_{0}, t_{0^{j} \uparrow_{0}}$ and $t_{0^{j-1}}$ are incompatible and $n_{0^{j-1}}$ is also in the illfounded part of $<_{w}$ (recall $n_{s}$ is the integer such that $u_{s}=\left(x_{n_{s}}\right)_{m_{s}}$ for some $m_{s}$ ). More generally, for each node $s \in 2^{<\omega}$ so that $n_{s}$ is in the illfounded part of $<_{w}$, the same argument shows that for some $s^{\prime} \supseteq s, t_{s^{\prime}-0}$ and $t_{s^{\prime} \wedge 1}$ are incompatible. Thus the tree $T_{z}$ whose set of branches constitute the set $\left(B_{0}^{n, m}\right)_{z}$ contains a perfect subtree, and therefore the set $\left(B_{0}\right)_{z}$ contains a perfect subset.

Finally we define our Borel set $B \subseteq \omega^{\omega} \times \omega^{\omega}$ with the required properties of the theorem. For $(z, y) \in \omega^{\omega} \times \omega^{\omega}$ put $(z, y) \in B$ iff

$$
(z \notin A \text { and } \forall i y(i)<2) \text { or }\left(z \in A \text { and } y \in S_{z}\right) \text { or }(z, y) \in B_{0} \text {. }
$$

It is easy to see that $B$ is Borel and has $K_{\sigma}$ sections. If $C$ is a small countable set, then for the unique $z \in A$ with $C=S_{z}$ we have that $B_{z}=C$ since $\left(B_{0}\right)_{z} \subseteq C$. If $z \notin A$, then $B_{z}$ is compact but perfect. If $z=\langle w, k, x\rangle \in A$ but $S_{z}$ is not small, then $<_{w}$ is illfounded and $\left(B_{0}\right)_{z}$ is uncountable, and therefore $B_{z}$ is again uncountable.

In a parallel development we show that large countable sets can also be uniquely represented, assuming $V=L$. The proof is a combination of the basic techniques we have been using in the proofs so far. 
Theorem 4.15. Assume $V=L$. Then there is a Borel set $B \subseteq \omega^{\omega} \times \omega^{\omega}$ such that

(a) all sections of $B$ are $K_{\sigma}$,

(b) all large countable sets are uniquely represented, and

(c) every section of $B$ is either a large countable set or else uncountable.

Proof. We will be overloading some notation from previous definitions. Since they will be used only in this proof there is no danger of confusion. First note that the set Tp of all pruned, perfect trees on $\omega$ is a Borel subset of $2^{\omega^{<\omega}}$. Thus we can fix a Borel bijection $z \mapsto T_{z}$ from $\omega^{\omega}$ onto this set of trees. Similarly there is a Borel bijection $z \mapsto S_{z}$ from $\omega^{\omega}$ onto the set Tr of all trees on $\omega$.

Let $<$ be a good $\Delta_{2}^{1}$ wellordering of the reals and let

$$
\begin{aligned}
R(x, z) \Leftrightarrow & \forall x^{\prime}<x\left(C\left(x^{\prime}\right) \neq C(x)\right) \wedge\left(C(x) \cap\left[T_{z}\right] \text { is dense in }\left[T_{z}\right]\right) \\
& \wedge \forall z^{\prime}<z\left(C(x) \cap\left[T_{z^{\prime}}\right] \text { is not dense in }\left[T_{z^{\prime}}\right]\right) .
\end{aligned}
$$

Then $R$ is $\Sigma_{2}^{1}$. By $\Pi_{1}^{1}$ uniformization as before there is $P \subseteq \omega^{\omega}$ which is $\Pi_{1}^{1}$ such that

$$
R(x, z) \leftrightarrow \exists w P(\langle x, z, w\rangle) \Leftrightarrow \exists ! w P(\langle x, z, w\rangle) .
$$

Since the set WF of all wellfounded trees on $\omega$ is $\Pi_{1}^{1}$-complete, there is a continuous reduction $f$ from $P$ to WF. It follows that there is a Borel function $u \mapsto S_{u}^{*}=S_{f(u)}$ from $\omega^{\omega}$ into Tr such that $P(u)$ iff $S_{u}^{*}$ is wellfounded.

In the remaining part of the proof we will define a Borel set $B_{0} \subseteq \omega^{\omega} \times \omega^{\omega}$ with $K_{\sigma}$ sections. The construction will ensure that, if $P(u)$ and $u=\langle x, z, w\rangle$, then $\left(B_{0}\right)_{u} \subseteq C(x)$, and if $\neg P(u)$, then $\left(B_{0}\right)_{u}$ is uncountable. Eventually we will define the required $B$ by

$$
(u, y) \in B \Longleftrightarrow(u, y) \in B_{0} \vee(u=\langle x, z, w\rangle \wedge y \in C(x)) .
$$

Then $B$ is Borel and all sections of $B$ are $K_{\sigma}$. If $C$ is a large countable set, then there is a unique $u=\langle x, z, w\rangle \in P$ such that $C=C(x)$. In this case $S_{u}^{*}$ is wellfounded, and this will guarantee that $\left(B_{0}\right)_{u} \subseteq C(x)$. It follows that $C=B_{u}$. If $u \notin P$, then $S_{u}^{*}$ is illfounded, and this will guarantee $\left(B_{0}\right)_{u}$ is uncountable and therefore $B_{u}$ is uncountable. If $u_{1}=\left\langle x_{1}, z_{1}, w_{1}\right\rangle$ and $u_{2}=\left\langle x_{2}, z_{2}, w_{2}\right\rangle$ are both in $P$ and $C\left(x_{1}\right)=C\left(x_{2}\right)$, then indeed $u_{1}=u_{2}$. To put all these together, we get that each large countable set is uniquely represented by $B$ and each countable section of $B$ is large.

Thus it remains to define the Borel set $B_{0}$. For this we work in a slightly more general context and prove the following abstract lemma.

Lemma 4.16. There is a Borel function

$$
g:\left\{(S, x, T) \in \operatorname{Tr} \times \omega^{\omega} \times \operatorname{Tp} \mid C(x) \subseteq[T] \text { and is dense in }[T]\right\} \rightarrow \operatorname{Tr}
$$

such that, for any $(S, x, T) \in \operatorname{dom}(g)$,

(1) $g(S, x, T)$ is finite-splitting,

(2) if $S$ is wellfounded, then $[g(S, x, T)] \subseteq C(x)$, and

(3) if $S$ is illfounded, then $g(S, x, T)$ contains a perfect subtree.

Proof. Suppose $S, x$ and $T$ are given. It is easy to modify $S$ so that if it is not wellfounded, then it contains a perfect subtree; for example, replace $S$ by the tree 
$S^{\prime}=\left\{\left(n_{0}, n_{1}, \ldots, n_{k}\right):\left(n_{0}, n_{2}, \ldots, n_{2\lfloor k / 2\rfloor}\right) \in S\right\}$. Also we assume without loss of generality that $S$ has the following property: for any $\tau \in \omega^{<\omega}$ if there is $n \in \omega$ such that $\tau^{\frown} n \in S$, then for all $n \in \omega$ we have $\tau^{\frown} n \in S$. For example, replace $S$ by $S^{\prime}=\left\{\left(n_{0}, n_{1}, \ldots, n_{k}\right):\left(n_{0}, \ldots, n_{k-1}\right) \in S\right\}$. These operations are Borel and do not affect the wellfoundedness of $S$.

Fix a continuous function $\tau \mapsto s_{\tau}$ from $S$ into $2^{<\omega}$ given by: if $\tau=\left(n_{0}, n_{1}, \ldots\right.$, $\left.n_{k}\right)$, then

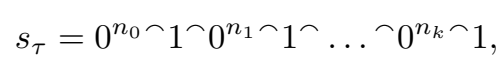

where $0^{i}$ denotes the sequence of $i$ many 0 's. Let $S^{*}$ be the subtree of $2^{<\omega}$ generated by the set $\left\{s_{\tau} \mid \tau \in S\right\}$.

We next define, by induction on $s \in 2^{<\omega}$, the following: $u_{s} \in C(x), l_{s} \in \omega$, $t_{s} \in T$, and eventually let $g(S, x, T)$ be generated by $\left\{t_{s}\right\}_{s \in 2<\omega}$. In fact, we let $t_{s}=u_{s} \uparrow l_{s}$, so it remains to define $u_{s}, l_{s}$. To begin the definition, let $u_{0^{i}}=x_{0}$ for all $i \in \omega$. In particular $u_{\emptyset}=x_{0}$. If $\langle 0\rangle \notin S^{*}$, then make arbitrary definitions of all $t_{s}$ so that $t_{s} \subseteq u_{\emptyset}$ and $t_{s} \subseteq t_{s^{\prime}}$ when $s \subseteq s^{\prime}$. Then $g(S, x, T)$ is trivially finite-splitting, $S$ is trivially wellfounded and $[g(S, x, T)]=\left\{x_{0}\right\} \subseteq C(x)$. If $\langle 0\rangle \in S^{*}$, then let $m>0$ be the smallest natural number such that for some $l>0, x_{m} \uparrow l=x_{0} \uparrow l$. Such an $m$ can be found since $C(x)$ is dense in $[T]$. Then let $u_{\langle 0 \wedge 1\rangle}=x_{m}$ and $l_{\emptyset}$ be the largest such that $u_{\langle 0-1\rangle} \uparrow l_{\emptyset}=u_{\langle 0\rangle} \uparrow l_{\emptyset}$.

To continue we inductively define $l_{0^{i}}$ and $u_{0^{i} 1}$ for all $i$. Note that if $\langle 0\rangle \in S^{*}$, then $0^{i} \in S^{*}$ for all $i$. The case $i=0$ has been done above. In general suppose $l_{0^{i}}$ and $u_{0^{i}-1}$ have been defined. Let $M=\left\{x_{m} \mid x_{m}=u_{0^{j} 1}\right.$ for some $\left.j \leq i\right\}$. Let $m \notin M$ be the smallest natural number such that for some $l>l_{0^{i}}, x_{m}\left\lceil l=x_{0^{i}}\lceil l\right.$.

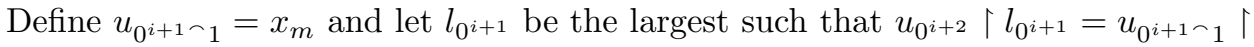
$l_{0^{i+1}}$.

Now the general inductive definition for $u_{s}$ and $l_{s}$ can be made in the same fashion as above, with $s$ replacing $\emptyset$. More precisely, for $s$ ending with 1 , let $u_{s^{\frown} 0^{i}}=u_{s}$. If $s^{\frown} 0 \notin S^{*}$, then make arbitrary definitions of $t_{s^{\prime}}$ for $s \subseteq s^{\prime}$ so that $t_{s^{\prime}} \subset u_{s}$ and $t_{s^{\prime}} \subset t_{s^{\prime \prime}}$ when $s^{\prime} \subset s^{\prime \prime}$. If $s^{\frown} 0 \in S^{*}$ is defined, then inductively define $l_{s \frown 0^{i}}$ and $u_{s \frown 0^{i}{ }_{1}}$ for all $i$ in the same fashion as we defined $l_{0^{i}}$ and $u_{0^{i}{ }_{1}}$. This finishes the definition of the sequences $u_{s}, l_{s}$ and $t_{s}$.

Since $t_{s} \subset t_{s^{\prime}}$ when $s \subset s^{\prime}$, the tree $g(S, x, T)$ generated by $\left\{t_{s}\right\}_{s \in 2<\omega}$ is well defined. It is tedious but straightforward to see that $g$ is a Borel function. Also it is clear that $g(S, x, T)$ is finite-splitting, since each $t_{s}$ has at most two immediate descendants, namely, $t_{s \frown 0}$ and $t_{s\urcorner 1}$. It remains to verify clauses $(2)$ and (3) of the lemma.

To verify (2), suppose $S$ is wellfounded. In this case the only infinite branches of $S^{*}$ are the sequences which are eventually 0's. Thus each infinite branch of $S^{*}$ corresponds to some $s \in 2^{<\omega}$ where $t_{s^{\prime}} \subset u_{s}$ for all $s \subset s^{\prime}$. It follows from our construction that $[g(S, x, T)] \subseteq\left\{u_{s} \mid s \in 2^{<\omega}\right\} \subseteq C_{x}$.

To verify (3), suppose $S$ is illfounded. By our hypothesis $S$ contains a perfect subtree. For notational simplicity assume that $S$ itself is perfect. We claim that the set $\left\{t_{s} \mid s \in S^{*}\right\}$ generates a perfect subtree of $g(S, x, T)$. To see this, let $s \in S^{*}$.

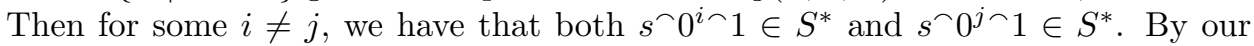
construction, $t_{s \frown 0^{i+1} \frown 1}$ and $t_{s^{\frown} 0^{j+1} \frown 1}$ are incompatible. This finishes the proof of the lemma. 
Now to complete the proof of the theorem, simply let

$$
(u, y) \in B_{0} \Longleftrightarrow y \in\left[g\left(S_{u}, x^{*}, T_{z}\right)\right],
$$

where $u=\langle x, z, w\rangle$ and $x \mapsto x^{*}$ is a Borel function such that $x^{*}=x$ if $C(x) \cap\left[T_{z}\right]$ is dense in $T_{z}$, and otherwise $C\left(x^{*}\right) \subseteq\left[T_{z}\right]$ is dense. Then $B_{0}$ has all the desired properties.

Note that for the Borel set just constructed each section is in fact a countable union of sets, all but one are singletons and the remaining one is compact. A technical curiosity is whether one can do the same for the Borel set uniquely representing small countable sets. We do not know the answer to this question.

Putting the Borel sets constructed in the previous two theorems side by side, we obtain a Borel set with $K_{\sigma}$ sections which uniquely represents all countable sets. That is, let $B_{0}$ be as in theorem 4.5 and $B_{1}$ as in theorem 4.15. Then let

$$
B(x, y) \Leftrightarrow\left(x(0)>1 \wedge y \in 2^{\omega}\right) \vee\left(x(0)=0 \wedge B_{0}(\bar{x}, y)\right) \vee\left(x(0)=1 \vee B_{1}(\bar{x}, y)\right),
$$

where $\bar{x}(n)=x(n+1)$. This completes the proof of theorem 4.1.

\section{UNIVERSAL SETS FOR $\boldsymbol{\Delta}_{\alpha}^{0}$ SETS}

As we mentioned before, results of [8], [11] show that there is no analytic set which is universal for the countable $\boldsymbol{\Delta}_{2}^{0}$ sets (which are the same as the scattered sets by proposition 4.3). As for the $\boldsymbol{\Delta}_{2}^{0}$ sets in general, we show that there is an analytic set, but not a Borel set, which is universal for the $\boldsymbol{\Delta}_{2}^{0}$ sets. In other words, $R\left(\boldsymbol{\Delta}_{1}^{1}, \boldsymbol{\Delta}_{2}^{0}, \boldsymbol{\Delta}_{2}^{0}\right)$ fails but $R\left(\boldsymbol{\Sigma}_{1}^{1}, \boldsymbol{\Delta}_{2}^{0}, \boldsymbol{\Delta}_{2}^{0}\right)$ holds. We then generalize this to higher levels of the Borel hierarchy.

We first show the easy result that $R\left(\boldsymbol{\Sigma}_{1}^{1}, \boldsymbol{\Delta}_{2}^{0}, \boldsymbol{\Delta}_{2}^{0}\right)$ holds in the following proposition.

Proposition 5.1. There is an analytic set in $X \times Y$ with all sections $\boldsymbol{\Delta}_{2}^{0}$ and which represents all the $\boldsymbol{\Delta}_{2}^{0}$ subsets of $Y$.

Proof. By remark 1.6 we may assume $X=\omega^{\omega}$. As a special instance of our coding of Borel sets, we view every $x \in \omega^{\omega}$ as coding a tree $T_{x}$ of height 2 which then determines a $\boldsymbol{\Sigma}_{2}^{0}$ set $D(x) \subseteq Y$. Define then

$$
A(x, y) \Leftrightarrow \exists z\left[z \in\left(D\left(x_{0}\right) \cap D\left(x_{1}\right)\right) \vee z \notin\left(D\left(x_{0}\right) \cup D\left(x_{1}\right)\right)\right] \vee\left(y \in D\left(x_{0}\right)\right) .
$$

This easily works.

We say that $x \in \omega^{\omega}$ is a $\boldsymbol{\Delta}_{2}^{0}$ code (with respect to the Polish space $Y$ ) if, in the above notation, $D\left(x_{0}\right)=Y \backslash D\left(x_{1}\right)$. In this case, $x$ codes the $\boldsymbol{\Delta}_{2}^{0}$ set $D\left(x_{0}\right)$. The previous proof was basically just the observation that the set of $\boldsymbol{\Delta}_{2}^{0}$ codes is a $\boldsymbol{\Pi}_{1}^{1}$ set.

We next show the negative result. We first review some facts about $\boldsymbol{\Delta}_{2}^{0}$ sets that we need. If $B \subseteq Y$ is $\boldsymbol{\Delta}_{2}^{0}$ and $F \subseteq Y$ is closed, then it cannot be the case that both $B$ and $Y \backslash B$ are dense in $F$ (the intersection of two dense $G_{\delta}$ 's in the Polish space $F$ must be dense). Hence if we let $U=U(F, B)$ be the union of all basic open sets $N_{i}$ in $Y$ such that $N_{i} \cap F \subseteq B$ and $V=V(F, B)$ be the union of all basic open sets $N_{i}$ in $Y$ such that $N_{i} \cap F \subseteq Y \backslash B$, then $F \cap(U \cup V) \neq \emptyset$. Define the resolvable derivative by $D_{B}(F)=F \backslash(U \cup V)$. So, for closed $F, D_{B}(F) \subsetneq F$ and $D_{B}(F)$ is also closed. Starting with $D_{B}^{0}=F$ and iterating this derivative (taking intersections at limits as usual), we define the $\alpha^{\text {th }}$ resolvable derivative $D_{B}^{\alpha}$. The 
resolvable derivative sequence must stop at a countable ordinal and for $B \in \boldsymbol{\Delta}_{2}^{0}$ we have shown this must stop at the empty set. We call the least $\alpha<\omega_{1}$ so that $D_{B}^{\alpha}=\emptyset$ the resolvable rank of $B$.

We can view the passage from $D_{B}^{\alpha}$ to $D_{B}^{\alpha+1}$ in two steps: first remove $U\left(D_{B}^{\alpha}, B\right)$, and then remove $V\left(D_{B}^{\alpha}, B\right)$. This defines a monotonically decreasing sequence of closed subsets $F_{\beta}$ of $Y$ of length $2 \cdot \alpha_{B}$, with $F_{0}=Y$ and with empty intersection, namely, $F_{2 \cdot \beta}=D_{B}^{\beta}, F_{2 \cdot \beta+1}=D_{B}^{\beta} \backslash U\left(D_{B}^{\beta}, B\right)$. Clearly $y \in B$ iff the least ordinal $\beta$ such that $y \notin F_{\beta}$ is odd. Recall that a set $B$ is said to be $\alpha-\Pi_{1}^{0}$ if there is an $\alpha$ length monotonically decreasing sequence of closed sets $F_{\beta}$ with empty intersection such that $y \in B$ iff the least $\beta$ such that $y \notin F_{\beta}$ is odd. We have thus proved the classical result of Hausdorff that $B \in \boldsymbol{\Delta}_{2}^{0}$ iff $B$ is $\alpha-\boldsymbol{\Pi}_{1}^{0}$ for some $\alpha<\omega_{1}$. The reader can consult $\S 37$ of [9] for a more detailed discussion.

In general, given a decreasing sequence of subsets $\left\{A_{\beta}\right\}_{\beta<\alpha}$ of a Polish space $Y$ with empty intersection, let $\mathcal{D}\left(\left\{A_{\beta}\right\}_{\beta<\alpha}\right)$ denote the corresponding difference set; that is, $y \in \mathcal{D}\left(\left\{A_{\beta}\right\}_{\beta<\alpha}\right)$ iff the least $\beta$ such that $y \notin A_{\beta}$ is odd.

By an operator $\mathcal{M}$ on a Polish space $X$ we mean a function $\mathcal{M}: \mathcal{P}(X) \rightarrow \mathcal{P}(X)$. We say $\mathcal{M}$ is monotone if whenever $A \subseteq B$, then $\mathcal{M}(A) \subseteq \mathcal{M}(B)$. We say $\mathcal{M}$ is a $\boldsymbol{\Pi}_{1}^{1}$ operator if the relation $x \in \mathcal{M}(A)$ is $\Pi_{1}^{1}(x, A)$, where the notion of being $\boldsymbol{\Pi}_{1}^{1}(x, A)$ is defined in a natural way (the precise definition is given in [3]). Any monotone operator $\mathcal{M}$ gives rise to an increasing sequence of subsets of $X$ defined by $E^{0}=\emptyset, E^{\alpha+1}=\mathcal{M}\left(E^{\alpha}\right)$, and $E^{\alpha}=\bigcup_{\beta<\alpha} E^{\beta}$ for the limit $\alpha$. The closure ordinal of $\mathcal{M}$ is the least $\alpha_{\mathcal{M}}$ such that $E^{\alpha_{\mathcal{M}}}=E^{\alpha_{\mathcal{M}+1}}$, and the least fixed point is $E(\mathcal{M}):=E^{\alpha \mathcal{M}}$. A main result of [3] says that if $\mathcal{M}$ is a $\Pi_{1}^{1}$ operator on a Polish space, then the least fixed point $E(\mathcal{M})$ is $\boldsymbol{\Pi}_{1}^{1}$, the closure ordinal $\alpha_{\mathcal{M}}$ satisfies $\alpha_{\mathcal{M}} \leq \omega_{1}$, and the following boundedness principle holds: if $A \subseteq E(\mathcal{M})$ is $\Sigma_{1}^{1}$, then there is an $\alpha<\omega_{1}$ such that $A \subseteq E^{\alpha}(\mathcal{M})$.

Theorem 5.2. There is no Borel set $B \subseteq X \times Y$ with all sections $\boldsymbol{\Delta}_{2}^{0}$ which represents all the $\boldsymbol{\Delta}_{2}^{0}$ subsets of $Y$.

Proof. Suppose $B \subseteq X \times Y$ is a Borel set with all sections $\boldsymbol{\Delta}_{2}^{0}$. We show that there is a bound $\alpha<\omega_{1}$ on the resolvable ranks of the sections of $B$. This shows that $B$ cannot be universal for the $\boldsymbol{\Delta}_{2}^{0}$ sets as the resolvable ranks of $\boldsymbol{\Delta}_{2}^{0}$ sets are unbounded in $\omega_{1}$. We can show this boundedness claim either by a direct computation along the lines of [8] or by appealing to the theory of $\boldsymbol{\Pi}_{1}^{1}$ monotone operators as presented in [3] and reviewed above. For variety, we take the second approach.

Consider the operator $\mathcal{M}$ on the Polish space $X \times Y$ defined by

$$
\begin{aligned}
(x, y) \in \mathcal{M}(A) \Leftrightarrow & (x, y) \in A \vee \exists i \in \omega\left\{\left(y \in N_{i}\right) \wedge\right. \\
& \left(\forall z \in Y\left[\left(z \in N_{i} \wedge(x, z) \notin A\right) \rightarrow(x, z) \in B\right]\right. \\
& \left.\left.\vee \forall z \in Y\left[\left(z \in N_{i} \wedge(x, z) \notin A\right) \rightarrow(x, z) \notin B\right]\right)\right\} .
\end{aligned}
$$

By inspection $\mathcal{M}$ is a $\boldsymbol{\Pi}_{1}^{1}$ operator, using the fact that $B$ is Borel, and it is easily monotone. $\mathcal{M}$ acts on each $x$-section of the product space $X \times Y$ separately, and for every $x \in X$, the restricted operator $\mathcal{M}_{x}$ builds up the sets $E_{x}^{\alpha}=Y \backslash D_{x}^{\alpha}$ where $D_{x}^{\alpha}$ is the $\alpha^{\text {th }}$ resolvable derivative of the section $B_{x}$ (so all sections of any $E^{\alpha}$ are open in $Y$ ). The closure ordinal of $\mathcal{M}_{x}$ is just the resolvable rank of $B_{x}$. Since all sections of $B$ are $\boldsymbol{\Delta}_{2}^{0}$, the least fixed point of $\mathcal{M}$ is the entire space $X \times Y$. Since this is a Borel set, the boundedness principle for $\Pi_{1}^{1}$ monotone operators mentioned 
above implies that the closure ordinal $\alpha_{\mathcal{M}}$ of $\mathcal{M}$ is countable. Clearly $\alpha_{\mathcal{M}}$ bounds the resolvable ranks of all the sections of $B$.

The technique of enlarging the Polish topology to make certain Borel sets clopen has many applications; see, for example, [7]. One application is to extend the difference hierarchy result mentioned above to higher levels. This gives the following well-known result, whose proof we sketch since we need it.

Lemma 5.3. Let $\alpha<\omega_{1}$. Then a set $A \subseteq Y$ is $\boldsymbol{\Delta}_{\alpha+1}^{0}$ iff it is in the difference hierarchy over $\boldsymbol{\Pi}_{\alpha}^{0}$, that is, there is a decreasing sequence $\left\{A_{\beta}\right\}_{\beta<\gamma} \subseteq \Pi_{\alpha}^{0}$ (for some countable ordinal $\gamma$ ) with $A=\mathcal{D}\left(\left\{A_{\beta}\right\}_{\beta<\gamma}\right)$.

Proof. Let $A \in \Delta_{\alpha+1}^{0}$. Write $A=\bigcup_{n} A_{n}$, and $B:=Y \backslash A=\bigcup_{n} B_{n}$, where $A_{n}, B_{n} \in \boldsymbol{\Pi}_{\alpha}^{0}$. Let $\tau$ denote the original Polish topology on $Y$. Let $\tau^{\prime} \supseteq \tau$ be the canonical larger Polish topology making all the $A_{n}, B_{n}$ closed (see [7]). Thus $A$ is $\Delta_{2}^{0}$ in $\tau^{\prime}$. Let $\left\{A_{\beta}\right\}_{\beta<\gamma}$ be a decreasing sequence of $\tau^{\prime}$ closed sets with $A=\mathcal{D}\left(\left\{A_{\beta}\right\}_{\beta<\gamma}\right)$. Each closed set in $\tau^{\prime}$, however, is $\boldsymbol{\Pi}_{\alpha}^{0}$ in $\tau$, so we are done.

The point we need to observe is that from Borel codes for $A$ and $Y \backslash A$ we can effectively describe the basic open sets in $\tau^{\prime}$.

If $A \subseteq Y$ is $\Delta_{\alpha+1}^{0}$, the resolvable rank of $A$ will denote the least $\alpha<\omega_{1}$ such that there is a decreasing sequence $\left\{A_{\beta}\right\}_{\beta<\alpha} \subseteq \boldsymbol{\Pi}_{\alpha}^{0}$ with $A=\mathcal{D}\left(\left\{A_{\beta}\right\}_{\beta<\alpha}\right)$. The resolvable ranks of the $\boldsymbol{\Delta}_{\alpha+1}^{0}$ sets are unbounded in $\omega_{1}$ by the same arguments as for $\Delta_{2}^{0}$.

We now combine and generalize proposition 5.1 and theorem 5.2 into the following.

Theorem 5.4. Let $\alpha<\omega_{1}$. Then there is an analytic, but not a Borel, set $B \subseteq$ $X \times Y$ which is universal for the $\boldsymbol{\Delta}_{\alpha+1}^{0}$ sets.

Proof. The existence of an analytic $B$ follows just as in proposition 5.1, now using a coding $x \rightarrow B(x)$ of $\boldsymbol{\Delta}_{\alpha+1}^{0}$ sets and the fact that the relation $B(y)=Y \backslash B(x)$ is still $\Pi_{1}^{1}$.

Suppose towards a contradiction that there were a Borel $B \subseteq X \times Y$ universal for the $\boldsymbol{\Delta}_{\alpha+1}^{0}$ subsets of $Y$. Again, we employ the coding $w \rightarrow D(w)\left(w \in \omega^{\omega}\right)$ of the $\boldsymbol{\Delta}_{\alpha+1}^{0}$ subsets of $Y$ obtained as a specialization of the Borel coding discussed in the introduction, using now just trees of height $\alpha+1$. We call $w \in \omega^{\omega}$ a $\boldsymbol{\Delta}_{\alpha+1}^{0}$ code for $D(w)$.

First note that there is a Borel function $f: X \rightarrow \omega^{\omega}$ such that for all $x \in X$, $f(x)$ is a $\boldsymbol{\Delta}_{\alpha+1}^{0}$ code for the set $B_{x} \subseteq Y$. To see this, first suppose without loss of generality that $B \in \Delta_{1}^{1}$. By Louveau's theorem [10], for every $x \in X$ there is a $\Delta_{1}^{1}(x)$ real $z \in \omega^{\omega}$ which is a $\Delta_{\alpha+1}^{0}$ code for $B_{x}$. Consider the relation

$$
R(x, w) \Leftrightarrow\left(w \in \Delta_{1}^{1}(x)\right) \wedge\left(w \text { is a } \Delta_{\alpha+1}^{0} \text { code }\right) \wedge\left(D(w)=B_{x}\right) .
$$

$R$ is $\boldsymbol{\Pi}_{1}^{1}$ and so has a $\boldsymbol{\Pi}_{1}^{1}$ uniformization $R^{\prime}$. Then $R^{\prime}$ is actually Borel since

$$
\neg R^{\prime}(x, w) \Leftrightarrow \exists z \in \Delta_{1}^{1}(x)\left[R^{\prime}(x, z) \wedge(z \neq w)\right],
$$

which is also $\Pi_{1}^{1}$ by bounded quantification. Clearly $R^{\prime}$ is then the graph of a Borel function $f$.

From $x \in X$ we can in a uniformly Borel manner compute $f(x)$ and then $\left\{N_{i}^{x}\right\}_{i \in \omega}$, which is a base for the topology $\tau_{x}:=$ the canonical enlargement of the 
given Polish topology $\tau$ on $Y$ which makes $B_{x}$ a $\boldsymbol{\Delta}_{2}^{0}$ set (as in lemma 5.3). Note that $\tau_{x}$ is canonically defined from the Borel code $f(x)$ for $B_{x}$. We now consider the operator $\mathcal{M}$ on $X \times Y$ defined exactly as in the proof of theorem 5.2 except we replace the $N_{i}$ there with $N_{i}^{x}$. The operator $\mathcal{M}$ is still a $\Pi_{1}^{1}$ operator since the relation $A(x, z, i) \leftrightarrow z \in N_{i}^{x}$ is Borel. As in theorem 5.2 we get an $\alpha_{\mathcal{M}}<\omega_{1}$ which bounds the resolvable ranks of all the $B_{x}$ as $\boldsymbol{\Delta}_{2}^{0}$ sets in the $\tau_{x}$ topology, and hence bounds their resolvable ranks as $\boldsymbol{\Delta}_{\alpha+1}^{0}$ sets. This is a contradiction since the resolvable ranks of the $\boldsymbol{\Delta}_{\alpha+1}^{0}$ sets are unbounded in $\omega_{1}$.

\section{REFERENCES}

[1] H. Becker, Borel and Analytic One-One Parametrizations of the Countable Sets of Reals, Proc. of the A.M.S., Vol. 103, Number 3 (1988), 929-932. MR947685 (89i:03091)

[2] H. Becker and A. S. Kechris, The Descriptive Set Theory of Polish Group Actions, London Mathematical Society Lecture Note Series 232, Cambridge University Press, Cambridge, 1996. MR1425877 (98d:54068)

[3] D. Cenzer and R. D. Mauldin, Inductive definability: Measure and category, Adv. in Math. 38 (1980), 55-90. MR594994 (82b:03086)

[4] R. Dougherty, S. Jackson and A. S. Kechris, The Structure of Hyperfinite Borel Equivalence Relations, Transactions of the A.M.S., Vol. 341, Number 1 (1994), 193-225. MR1149121 (94c:03066)

[5] H. Friedman and L. Stanley, A Borel Reducibility Theory for Classes of Countable Structures, Journal of Symbolic Logic, Vol. 54 (1989) 894-914. MR1011177 (91f:03062)

[6] W. Hurewicz, Zur theorie der Analytischen Mengen, Fundamenta Mathematicae, vol. 15 (1930), 4-17.

[7] A. S. Kechris, Classical Descriptive Set Theory, Graduate Texts in Mathematics 156, Springer-Verlag, New York, 1995. MR1321597 (96e:03057)

[8] A. S. Kechris and D. A. Martin, A Note on Universal Sets for Classes of Countable $G_{\delta}$ 's, Mathematika 22 (1975), 43-45. MR0420579 (54:8593)

[9] K. Kuratowski, Topology. Volume 1, Academic Press, New York and London, 1966. MR0217751 (36:840)

[10] A. Louveau, A Separation Theorem for $\Sigma_{1}^{1}$ Sets, Transactions of the A.M.S., Vol. 260, Number 2 (1980), 363-378. MR574785 (81j:04001)

[11] R. D. Mauldin, The boundedness of the Cantor-Bendixson order of some analytic sets, Pacific J. Math. 74 (1978), 167-177. MR0474236 (57:13883)

[12] S. Mazurkiewicz And W. Sierpinski, Sur un Problème Concernant les Fonctions Continues, Fundamenta Mathematicae, vol. 6 (1924), 161-169.

[13] Y. N. Moschovakis, Descriptive Set Theory, North-Holland Publ. Co., New York, 1980. MR561709 (82e:03002)

[14] J. Saint-Raymond, Boréliens à coupes $K_{\sigma}$, Bull. Soc. Math. France 104 (1976), no. 4, 389400. MR0433418 (55:6394)

Department of Mathematics, P.O. Box 311430, University of North Texas, Denton, TEXAS 76203

E-mail address: sgao@unt.edu

Department of Mathematics, P.O. Box 311430, University of North Texas, Denton, TEXAs 76203

E-mail address: jackson@unt.edu

Department of Analysis, Eötvös Loránd University, Budapest, Kecskeméti u. 10-12, HUNGARY 1053

E-mail address: laczk@cs.elte.hu

Department of Mathematics, P.O. Box 311430, University of North Texas, Denton, TEXAS 76203

E-mail address: mauldin@unt.edu 